\title{
Deformable Ultrasound Registration without Reconstruction
}

\author{
Rupert Brooks ${ }^{1}$, D. Louis Collins ${ }^{2}$, Xavier Morandi $^{3}$, and Tal Arbel ${ }^{1}$ \\ ${ }^{1}$ McGill University, Centre for Intelligent Machines, \\ rupert.brooks@mail.mcgill.ca, arbel@cim.mcgill.ca \\ ${ }^{2}$ McGill University, Dept. of Biomedical Engineering, \\ louis@bic.mni.mcgill.ca \\ ${ }^{3}$ University Hospital of Rennes, Dept. of Neurosurgery, \\ xavier.morandi@chu-rennes.fr
}

\begin{abstract}
Ultrasound (US) imaging is often proposed as an interoperative imaging modality. This use nearly always requires that the collected data be registered to preoperative data of another modality. Existing intensity-based registration approaches all begin by reconstructing a 3D US volume from the collected $2 \mathrm{D}$ slices. We propose to directly register the set of $2 \mathrm{D}$ slices to the preoperative images. We argue this has a number of advantages, including the omission of the potentially complex reconstruction step, greater adaptability of the similarity measures, and easier parallelization. We describe a system for performing this task and present results on phantom data that show that our slice based method consistently outperforms a reconstruction based method in both speed and accuracy.
\end{abstract}

\section{Introduction}

Ultrasound (US) imaging is used for a wide range of medical tasks ranging from diagnostic imaging to intra-operative image guidance. Many of these applications require the fusion of the ultrasound image with additional imagery in other modalities. As a motivating example, consider the use of $2 \mathrm{D}$ ultrasound imaging for interoperative guidance in neurosurgery. Preoperatively, a careful imaging study is often done and used for planning. During the procedure, however, the patient's brain deforms somewhat from its preoperative position. The US imagery can be used to track this deformation and update the preoperative information, but to accomplish this, it is necessary to register the interoperatively acquired images to the preoperative images. Unfortunately, registration of ultrasound data to other modalities has remained a challenging problem.

Previous approaches to the registration of $2 \mathrm{D}$ ultrasound data have as a first step reconstructed a 3D volume from the 2D slice data. This approach has several disadvantages. Volumetric reconstruction of US data is a challenging problem in its own right (see [1] for a review). It is also independent of the registration process, and so must be completed before the registration can begin. If problems are found with the reconstruction, or new data is acquired, the reconstruction must be reprocessed before the system can be updated. 
Instead, we propose to perform registration directly from a subset of the $2 \mathrm{D}$ ultrasound slices rather than reconstructing a volume. The most obvious advantage is that one does not have to go about the reconstruction process. Reconstruction takes some time and some data is inevitably lost in the resampling process. However, we believe the more important advantages of this approach arise from the separability of the similarity measure for each slice. This allows the registration process to be made more flexible in a number of ways. (1) The registration can be defined incrementally allowing new slices to be added, or faulty slices to be removed. (2) The similarity measures can be adjusted to the properties of the US on a slice by slice basis. This is important because as discussed in [2] it is difficult to find a reliable cost function for multimodal US registration. Treating the slices individually in this way gains some of the advantages of block-based local registration methods. (3) Working on a slice by slice basis also introduces opportunities for parallel computation, in both shared memory and non-shared memory contexts. The similarity measure for each slice can be processed by a separate core, or a separate process entirely. (4) Finally, it is possible to begin registration before all the slices are collected, which allows data collection to proceed in parallel with the optimization. This could lead to significant advantages in time critical environments.

Instead of a single, pairwise, registration between multimodal volumes, the registration without reconstruction strategy proposed in this paper is a groupwise registration problem expressed as the combination of $N$ pairwise slice to volume registrations. We have extended existing nonlinear registration algorithms to allow this groupwise registration and explore the performance of this approach on US and T1-MRI images of a deformable phantom object. We find that it is feasible and results outperform a volume reconstruction based approach.

In the following section we describe the proposed approach in more detail, and compare it to existing work. Section 3 describes our implementation which builds upon existing well tested deformable registration procedures. Our experimental data, procedure and results are described in Section 4.

\section{Methodology}

Parametric, intensity based, approaches to image registration work by defining a measure of similarity, $S\left(I_{1}, I_{2}\right)$, between the images to be registered and specifying a parameterized space of transformations to explore [3]. In the pairwise expression of the problem, one image, called the moving image, is warped by this transform, which makes it a function of the transformation parameters. The similarity measure between the two images is then also a function of the parameters. As the optimal registration is considered to be the point of maximal similarity, the registration problem can be expressed as an optimization problem:

$$
\boldsymbol{\phi}_{\text {opt }}=\underset{\boldsymbol{\phi}}{\operatorname{argmax}}\left(S\left(I_{f}(\boldsymbol{x}), I_{m}\left(\boldsymbol{x}^{\prime}(\boldsymbol{\phi})\right)\right)-\lambda_{r} R(\boldsymbol{\phi})\right)
$$

Here, $I_{f}(\boldsymbol{x})$ and $I_{m}(\boldsymbol{x})$ are the fixed and moving images considered as functions over some space. $\boldsymbol{x}$ is a set of pixel positions in that space, and $\boldsymbol{x}^{\prime}(\boldsymbol{\phi})$ is this set 
of pixel positions warped by the transformation specified by the parameters, $\phi$. The last term, $R(\phi)$ is an optional regularizer on the transformation parameters, weighted by a multiplier, $\lambda_{r}[3] .(R(\phi)$ is defined so that small values are better.)

The literature holds relatively few examples of intensity based registration of ultrasound to other modalities. Existing approaches [2, 4, [5, 7] have two common properties. They all use a reconstruction process as a first step, and they all find that mutual information (MI) on the image intensities alone is not always effective for registration so another similarity measure must be found.

Slice to volume registration of US data is surprisingly rare. It is used in the calibration through registration approach of Blackall et al. [6], where they solve for US calibration parameters through registration of the slices to a phantom model. It is also used in the alignment by reconstruction approach of [8]. In [8] they reconstruct an ultrasound volume by iteratively registering the $2 \mathrm{D}$ slices to the current reconstruction and reconstructing the volume based on the registered slices. Slice to volume registration has been most aggressively pursued by the fMRI community to correct for patient motion between slice acquisitions. For example [9] presents an algorithm which jointly maps a collection of fMRI slices into a volume while simultaneously estimating the patients trajectory. The work that is probably closest to that presented here is that of Penney et al. [10], who performed rigid registration of sparse US slices to a CT volume of the liver.

\section{Implementation}

Our proposal requires registering multiple images at once, which makes this a groupwise rather than a pairwise approach. We therefore define our groupwise similarity measure, $S_{g}$, as the weighted average of the similarities between each $2 \mathrm{D}$ image and the preoperative volume

$$
S_{g}(\phi)=\frac{1}{N} \sum_{i=1}^{N} \lambda_{i} S_{i}\left(I_{f_{i}}(\boldsymbol{x}), I_{m}\left(\boldsymbol{x}^{\prime}(\boldsymbol{\phi})\right)\right)-\lambda_{r} R(\boldsymbol{\phi})
$$

Here we have treated the $N$ ultrasound slices as the fixed images, $I_{f_{i}}$, and the preoperative volume as the moving image, $I_{m}$.

We have used a registration approach quite similar to that in [11], extended to support groupwise, regularized registration. As in [11], the deformation is modeled using using a tensor product of B-Splines, and the similarity measure is an efficient Parzen-window implementation of MI. We also apply the same Quasi-Newton optimization algorithm to maximize our similarity function. A two level multiresolution technique was used for the optimization to help avoid local minima. Unlike [1], the same B-Spline transformation model was used at both pyramid levels. Also unlike [11], rather than using a B-Spline interpolation we used linear interpolation for efficiency reasons. We used a B-Spline resolution of $8^{3}$ nodes, of which $5^{3}$ are in the ROI, and the rest act as support.

The extension to support groupwise registration is straightforward. The similarity measure to be optimized is now the mean of the similarity measures for 
each slice (each slice was equally weighted). We also added a regularization term which approximates the linear elastic strain energy. This was computed using a $10^{3}$ grid of points spanning the ROI. Each point was considered to be connected to its 6 lattice neighbors and two diagonal neighbors with linear elastic springs. That is, each connection is considered to exert a force linearly proportional to its change in length. Spring energy being proportional to force squared, this gives rise to the following regularization term,

$$
\left.R(\phi)=\frac{1}{N} \sum_{n=1}^{N}\left(\| \boldsymbol{x}_{n_{1}}-\boldsymbol{x}_{n_{2}}\right)\|-\| \boldsymbol{x}_{n_{1}}^{\prime}\left(\phi_{0}\right)-\boldsymbol{x}_{n_{2}}^{\prime}\left(\phi_{0}\right) \|\right)^{2}
$$

where the summation is over all the connections. The same regularization was used for all registrations.

MI of the intensities was used for all MR-MR registrations. However, using MI directly for registering MR and US data may be unreliable [2]. For these experiments we computed MI between the gradient magnitudes of both images, a similarity measure similar to one used in [7]. Also as in [7], the US images were pre-smoothed with anisotropic diffusion [12]. Note that for the registration the $2 \mathrm{D}$ ultrasound gradient magnitude slice is matched to the $3 \mathrm{D}$ MRI gradient magnitude volume. However, for US volume reconstruction the gradient magnitude is first computed on the US slices which are then reconstructed into a volume. This was done because the reconstructed volume is somewhat sparse, making its gradient very noisy.

\section{Experiments}

The datasets used for this experiment consist of co-registered T1 MRI and tracked ultrasound images of a deformable phantom object. The phantom was constructed with an inflatable balloon embedded in it. By filling the balloon to varying degrees with water, a controlled deformation of the phantom was induced. It was scanned with both modalities at three levels of inflation, referred to here as deflated (D), partially inflated (P) and fully inflated (F). The MRI image has a resolution of $1 \mathrm{~mm}^{3}$, and the US slices have a resolution of approximately $0.4 \mathrm{~mm}$. Each US dataset contains an average of 46 slices. A region of interest (ROI) which had a large and very visible amount of deformation was defined, and our registration experiments were conducted over that ROI. This area and the deformations are illustrated in Figure 1.

For corresponding levels of inflation of the phantom, the MR and Ultrasound data had to be brought into the same reference frame. This was approximately achieved by a fiducial-based calibration during data collection and refined by rigid registration using the same algorithm described here. For these experiments, we have considered the ultrasound slices to be precisely in the same reference frame, and in the same frame as their corresponding MRI dataset. This is not truly the case, and we anticipate that this may cause some additional error in the registration experiments. 


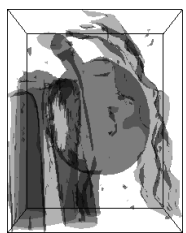

(a) Fully Inflated (F)

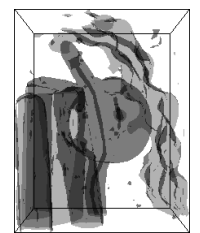

(b) Partially Inflated (P)

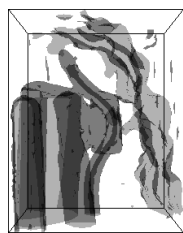

(c) Deflated (D)

Fig. 1. The region of interest for the registration experiments. These are 3D renderings of an isosurface at the middle intensity range showing the balloon phantom at each of the scanned levels of deformation.

To provide a synthetic experiment that will test our method without being affected by this error, we resampled the MRI datasets in the position of each ultrasound slice. This provides a set of synthetic slices that have extremely good intensity compatibility with the MRI data (limited only by the interpolation), and which are perfectly registered with their corresponding MRI dataset.

In order to compare with a volume to volume registration approach, volumes were reconstructed from both the ultrasound slices, and from the resampled MR slices. The volume was reconstructed by regridding with a 3D Gaussian kernel. To preserve detail, the slices were regridded into a $0.5 \mathrm{~mm}^{3}$ volume. In both cases, all available slices were used. (Note this does not simply reconstruct the original MR volume. The resulting volume is somewhat sparse because the US slices do not cover the space perfectly.)

Thus there are 6 deformation fields we can try to recover with our algorithm. We identify these as (D-P, D-F, P-D, P-F, F-D, F-P) where, for example, D-P refers to using the deflated case as the fixed image and the partially inflated case as the moving image. For each field there are 5 possible experiments, specifically:

1. (MR-V) The original MR volumes can be registered using volume registration. As this data is of good quality and contrast, results on this case probably represent the upper limit of performance.

2. (MR-S) Slice to volume registration can be performed on a selected set of MR slices. For this registration we selected 10 slices covering the ROI well.

3. (MR-R) A volume can be reconstructed from all the simulated MRI slices and registered using volume registration.

4. (US-S) A slice to volume registration can be performed on a selected set of US slices. The same 10 slices as in case MR-S were used.

5. (US-R) A volume can be reconstructed from all of the US slices and registered using volume registration.

We anticipate that the first case (MR-V) would give us the best possible accuracy under these conditions and provide kind of an upper bound for our work. Cases MR-S and MR-R should be directly comparable. These cases should test the slice to volume registration under synthetic conditions free from confounding factors. Finally, the last two cases US-S and US-R represent the realistic and difficult 
Table 1. mTRE in mm for the registrations by case and method. Cases in bold showed a statistically significant difference. Cases where the mTRE was reduced by at least $10 \%$ are underlined.

\begin{tabular}{c|ccccccc}
\hline Case & START & MR-V & MR-S & MR-R & US-S & US-R \\
\hline D-P & 2.25 & 2.35 & $\mathbf{2 . 1 4}$ & 2.26 & $\mathbf{2 . 1 6}$ & $\mathbf{2 . 1 5}$ \\
D-F & 2.69 & 2.69 & $\mathbf{2 . 1 3}$ & $\mathbf{2 . 5 1}$ & $\underline{\mathbf{2 . 0 4}}$ & $\mathbf{2 . 5 2}$ \\
P-D & 3.26 & $\underline{\mathbf{1 . 4 1}}$ & $\underline{\mathbf{1 . 2 1}}$ & $\underline{\mathbf{1 . 4 1}}$ & $\underline{\mathbf{2 . 0 9}}$ & $\underline{\mathbf{2 . 3 7}}$ \\
P-F & 2.39 & $\underline{\underline{\mathbf{0 . 9 5}}}$ & $\underline{\mathbf{0 . 9 4}}$ & $\underline{\mathbf{1 . 0 0}}$ & $\underline{\mathbf{1 . 9 6}}$ & $\mathbf{2 . 7 9}$ \\
F-D & 5.20 & $\underline{\mathbf{1 . 8 3}}$ & $\underline{\mathbf{2 . 2 2}}$ & 4.91 & $\underline{5.67}$ & $\mathbf{6 . 7 4}$ \\
F-P & 3.40 & $\underline{\mathbf{1 . 1 5}}$ & $\underline{\underline{\mathbf{1 . 2 1}}}$ & $\underline{\mathbf{1 . 2 7}}$ & $\underline{\mathbf{3 . 0 5}}$ & $\mathbf{4 . 6 2}$ \\
\hline
\end{tabular}

case that we are trying to solve. We anticipate that these cases will be the most difficult, as the slice to volume registration must also contend with the difficult US to MRI matching problem and any misalignment between individual slices, or between slices and their corresponding MRI image.

\section{$4.1 \quad$ Results}

Verification and presentation of the accuracy of a nonrigid transformation is a challenge. In this case, the MRI image has very good contrast and is reasonably easy to segment. We measured the accuracy by segmenting the structure of interest - in this case the balloon - as a dense mesh. Such a mesh was computed for each of the three MRI volumes. After a registration, the mesh of the fixed image was warped by the transformation. The distances between every point in this mesh and the nearest point in the moving image mesh were used as a measure of the registration accuracy. As these meshes are dense, with triangles on the order of the voxel size, and come from a single structure, we consider this a reasonable measure of the amount of deformation recovered.

Results for each case, in terms of the mean error, for each of the possible registration methods are shown in Table 1. A Mann-Whitney U-test (99 confidence level) was performed to determine where the error distributions are significantly different. Cases where the mTRE has been reduced by $10 \%$ or more are underlined. The results mostly follow the pattern that was expected. The MR-MR volume registrations give the best results, and probably give the upper limit of refinement of the deformation given the chosen registration framework. We see that in all cases the slice based registration outperforms the registrations based on reconstructed volumes, despite using significantly less data, even in the MR-MR case. This occurs for two reasons. The main reason is that the MI is computed independently for each slice giving the method more local adaptability. The second reason, more important on the ultrasound data, is that despite the fact that the reconstruction uses a fairly high resolution grid some blurring and data loss has inevitably taken place.

US-S achieves $10 \%$ or better improvement in 4 of 6 cases, while US-R achieves in only 3 of 6 cases. Of particular interest is the case D-F. This case is an 

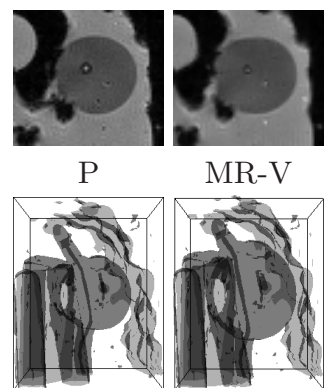

P

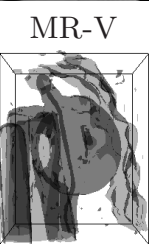

MR-V

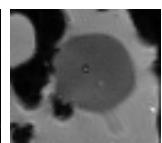

MR- $\mathrm{R}$

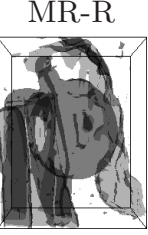

MR-R

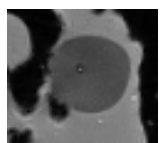

MR-S

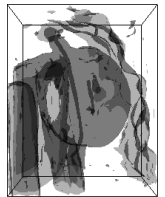

MR-S

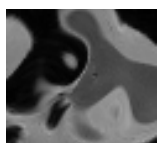

US-R

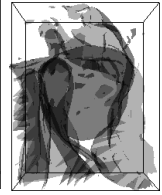

US-R

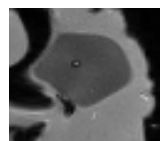

US-S

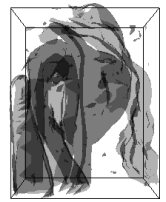

US-S

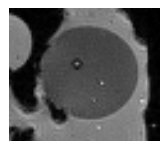

F

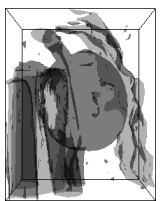

F

Fig. 2. Registration for Case P-F. The upper row shows cross sections through the middle of the ROI. In this case the registration should transform the image at the far right into the one on the far left. Conversely, the 3D rendering on the far left should be transformed into the one on the far right.
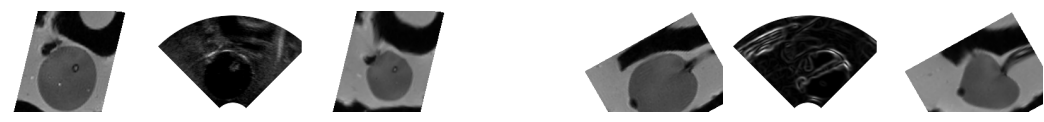

Fig. 3. Case P-F: Two typical US slices and the corresponding MR data. The MR data before registration is shown to the left, and that after registration to the right, of its US slice. The US slice in the left group is a plain B-Scan, and that in the right group is a gradient image.

extremely difficult registration, and the volumetric MR-MR registration fails on it. However, both slice based methods achieve some measure of success on this very difficult case. We believe this is because the flexibility of using a separate histogram for each slice helps the optimization escape a local minima.

It is difficult to describe the performance of a nonrigid transformation with one or two numbers. Here we will examine the case of registration of $\mathrm{P}-\mathrm{F}$ in detail. Figure 2 shows cross sections and 3D renderings of the original and registered versions for each case. The MR-V, MR-R, and MR-S cases produce good registrations, that are nearly identical in appearance. The US-S registration is obviously on the right track, with the volume of the balloon changing appropriately, but there remain clear imperfections. The US-R registration is a failure, with a bizarrely stretched result despite the presence of the elastic regularizer. Figure 3 shows corresponding MR and US images for two slices before and after registration for case US-S. Note the close fit of the balloon after registration.

Figure 4 shows histograms of the distances for the point to point matching between the meshes for each case. When starting out, the errors are distributed in a fairly flat manner. A successful registration should shift the bulk of the values to the left, and this is clearly shown for the MR-V, MR-S, and MR-R cases. The effect is present, but somewhat less dramatic for the case of US-S. The failed case, US-R, not surprisingly shows little to no improvement. 


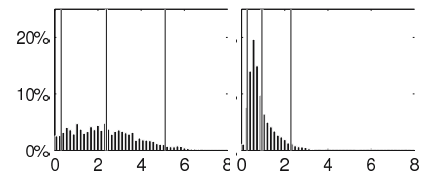

(a) START

(b) MR-V

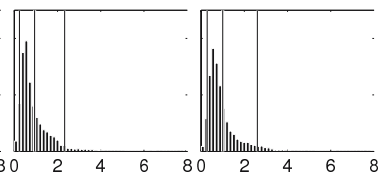

(c) MR-S (d) MR-R

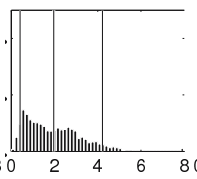

(e) US-S

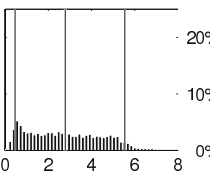

(f) US-R

Fig. 4. Histograms of residual distances for Case P-F. Vertical lines show the $5 \%$ percentile, the mTRE and the $95 \%$ percentile.

In terms of computational performance, our method was faster than the volume reconstruction method, even ignoring the time required to build the volume and considering only registration time. Running single threaded, our method averaged approximately 26 minutes to complete on a $2.4 \mathrm{GHz}$ Pentium 4 , while the volume based method took on average approximately 1.5 hours.

\section{Discussion}

This paper has presented a preliminary implementation of a method for registration of 2D ultrasound slices to a volume without a reconstruction step. Results on phantom data show that the method generally performs better in terms of both accuracy and speed when compared to a reconstruction oriented approach. More importantly, the method has a number of advantages, including the opportunity to add or remove slices easily, and opportunities for easy parallelization.

In future, we will expand the method to include a means of automatically selecting slices. We also plan to exploit some of the opportunities for parallelization to make possible near real-time approaches to parametric deformable registration. Finally, incorporating more sophisticated similarity functions for US-MRI matching (e.g. [2]) into this framework could lead to improved performance.

\section{References}

1. Solberg, O.V., et al.: Freehand 3d ultrasound reconstruction algorithms -a review. Ultrasound in Medicine and Biology 33(7), 991-1009 (2007)

2. Coupé, P.: Méthode de compensation des déformations cérébrales par imagerie ultrasonore intraopératoire pour la neurochirurgie guidée par l'image. $\mathrm{PhD}$ thesis, Université de Rennes (January 2008)

3. Modersitzki, J.: Numerical Methods for Image Registration. Numerical Mathematics and Scientific Computation. Oxford University Press, Oxford (2004)

4. Arbel, T., et al.: Automatic non-linear MRI-ultrasound registration for the correction of intra-operative brain deformations. In: Niessen, W.J., Viergever, M.A. (eds.) MICCAI 2001. LNCS, vol. 2208, pp. 913-922. Springer, Heidelberg (2001)

5. Roche, A., et al.: Rigid registration of 3D US with MR images: a new approach combining intensity and gradient information. IEEE Tr. Med. Imag. 20(10), 1038-1049 
6. Blackall, J.M., et al.: An image registration approach to automated calibration for freehand 3d ultrasound. In: Delp, S.L., DiGoia, A.M., Jaramaz, B. (eds.) MICCAI 2000. LNCS, vol. 1935, pp. 462-471. Springer, Heidelberg (2000)

7. Letteboer, M.M.J., et al.: Brain shift estimation in image-guided neurosurgery using 3d ultrasound. IEEE Transactions on Biomedical Engineering 52(2) (2004)

8. Sanches, J.M., Marques, J.S.: Joint image registration and volume reconstruction for 3d ultrasound. Pattern Recognition Letters 24, 791-800 (2003)

9. Park, H., Meyer, C., Kim, B.: Improved motion correction in fMRI by joint mapping of slices into an anatomical volume. In: Barillot, C., Haynor, D.R., Hellier, P. (eds.) MICCAI 2004. LNCS, vol. 3217, pp. 745-751. Springer, Heidelberg (2004)

10. Penney, G.P., et al.: Registration of freehand $3 \mathrm{~d}$ ultrasound and magnetic resonance liver images. Medical Image Analysis 8(1), 81-91 (2004)

11. Mattes, D., et al.: PET-CT image registration in the chest using free-form deformations. IEEE Trans. Medical Imaging 22(1), 120-128 (2003)

12. Perona, P., Malik, J.: Scale-space and edge detection using anisotropic diffusion. IEEE Trans. Pattern Analysis and Machine Intelligence 12(7) (1990) 